\title{
The Neuroprotective Effects of Danggui-Shaoyao San on Vascular Cognitive Impairment: Involvement of the Role of the Low-Density Lipoprotein Receptor-Related Protein
}

\author{
Haobin Cai, ${ }^{1}$ Tiantian Cai, ${ }^{2}$ Haotao Zheng,, Lijin Liu, ${ }^{1}$ Liuchang Zhou, ${ }^{1}$ Xile Pang, ${ }_{1}^{1}$ \\ Qinkai Zhan, ${ }^{2}$ Yijie Wang, ${ }^{2}$ Cong Yang, ${ }^{2}$ Zhouke Guo, ${ }^{1}$ Huafeng Pan, ${ }^{2}$ and Qi Wang ${ }^{2}$
}

\begin{abstract}
Effective drugs for treating dementia are still rare. Danggui-Shaoyao San (DSS), a traditional Chinese medicine, has been widely used in oriental countries for the treatment of various gynecological diseases. Many studies reported that DSS could ameliorate cognitive impairment. In this study, we aimed to investigate the underlying mechanism of DSS on vascular cognitive impairment (VCI) rats. Chronic cerebral hypoperfusion $(\mathrm{CCH})$ is one of the main causes of VCI. CCH resulted in a chain of pathological process, including neuroinflammation, neuronal apoptosis, and oxidative stress. The most widely used animal model of VCI is permanent bilateral common carotid artery occlusion in rats. In this research, we determined whether DSS attenuated cognitive impairment by targeting I kappa B kinase (IKK)/nuclear factor of kappa B (NF- $\kappa$ B) signal pathway in VCI rats. Morris water maze and fear conditioning tests results indicated that DSS $[7.2 \mathrm{~g} /(\mathrm{kg} \cdot \mathrm{d})]$ could improve learning and memory ability in VCI rats. We also found DSS significantly elevated the levels of low-density lipoprotein receptor-related protein 1 (LRP1) in the brain of VCI rats and this might indirectly target the IKK/NF- $\kappa \mathrm{B}$ signal pathway to exert inhibitory effect on neuroinflammation, neuronal apoptosis, and oxidative stress in VCI rats. The present researches indicated that DSS might attenuate cognitive impairment by targeting IKK/NF- $\kappa \mathrm{B}$ signal pathway in VCI rats and DSS might be a promising agent on VCI.
\end{abstract}

Keywords: vascular cognitive impairment, chronic cerebral hypoperfusion, Danggui-Shaoyao San, LRP1, IKK/ $\mathrm{NF}-\kappa \mathrm{B}$ signal pathway

\section{Introduction}

A MONG THE SUBTYPES OF DEMENTIA, vascular cognitive impairment (VCI) accounts for $15 \%-20 \%$ of all cases of dementia, ${ }^{1}$ which is not only the second common dementia after Alzheimer disease, but also a potential risk factor contributing to the development of Alzheimer's disease.,3

The clinical syndromes of VCI include cognitive dysfunction, emotional disorder, and diminish executive capacity. These symptoms have a negative impact on the daily life of patients with VCI. ${ }^{4}$ With the gradual increase in VCI patients, effective treatments are more and more urgent. Many studies have been devoted to the neuroinflammation and oxidative stress owing to chronic inflammation and long-term cerebrovascular dysfunction deficiency in cognitive altered process; these studies focused on endothelium and neuronal disruption acting as a promoter of neurodegenerative events. ${ }^{5}$ The present investigations focus on neuroprotective mechanisms of the central nervous system to reverse the pathological process of VCI.

Traditional Chinese medicine has been widely applied in preventing and treating diseases for 2000 years, and quantities of Chinese herbal medicine formulas are used to improve cognitive function. ${ }^{6,7}$ Danggui-Shaoyao San (DSS), a Chinese herbal compound, contains the following herbs: Angelica sinensis, Ligusticum chuanxiong Hort, Paeonia lactiflora Pall, Atractylodes macrocephala, Alisma Plantago-aquatica Linn, and Poria cocos, and it has been widely used in oriental

\footnotetext{
${ }^{1}$ Department of Neurology \& Psychology, Shenzhen Traditional Chinese Medicine Hospital, Fourth Clinical Medical College of Guangzhou University of Chinese Medicine, Shenzhen, China.

${ }^{2}$ Institute of Clinical Pharmacology, Guangzhou University of Chinese Medicine, Guangzhou, China.
} 
countries for the treatment of various gynecological diseases. ${ }^{8}$ It has been reported that DSS has an effect on free radicalmediated neurological diseases, exhibits anti-inflammatory and antioxidant activities, and reduces cell apoptosis in the hippocampus. ${ }^{9}$ However, mechanism of DSS on ameliorating the cognitive impairment in VCI still has not been reported. The dihydropyridinic calcium antagonist nimodipine has been proposed as a drug that is able to improve cognition in VCI because of vasoactive and neuroprotective actions. ${ }^{10}$ Its effect on age-related microangiopathy in experimental models ${ }^{11}$ makes nimodipine a potential treatment for small-vessel VCI subtypes.

Low-density lipoprotein (LDL) receptor-related protein 1 (LRP1) is a member of LDL receptor gene family, which clears $\beta$-amyloid $(\mathrm{A} \beta)$ peptides in the brain. ${ }^{12}$ Many investigations indicate that LRP1 inhibits $\mathrm{NF}-\kappa \mathrm{B}$ activity via down-regulating cell surface tumor necrosis factor receptor-1 (TNFR1). ${ }^{13}$ Several experimental studies have shown that oxidative stress and neuroinflammation play critical roles in VCI. ${ }^{14,15}$ In addition, I kappa B kinase (IKK) induced phosphorylation of $\mathrm{I} \kappa \mathrm{B}$, which promotes $\mathrm{I} \kappa \mathrm{B}$ degradation and release, finally leading to activation of NF- $\kappa \mathrm{B} .{ }^{16,17}$ IKK/NF- $\kappa \mathrm{B}$ pathway exerts antiapoptosis function through many other proteins, such as Bcl-2. It has also been shown that the levels of Bcl-2 protein are increased in neurons of vulnerable brain regions after global and local cerebral ischemia. ${ }^{18-22}$

Based on the previous study, we used the bilateral common carotid arteries occlusion (BCCAO) model to explore whether DSS could improve cognitive function through IKK/NF- $\kappa \mathrm{B}$ pathway (we explored the cognitive function of DSS through IKK/NF- $\kappa$ B pathway). ${ }^{23,24}$ The whole experimental process is given in Figure 1. Our experiment demonstrated the potential mechanism of DSS in ameliorating cognitive impairment in VCI through the IKK/NF- $\kappa \mathrm{B}$ pathway by regulated LRP1.

\section{Materials and Methods \\ Drugs}

The qualitative analysis of DSS formula had been performed in a previous research, ${ }^{25}$ which contains the following ingredients: Angelica sinensis, Ligusticum chuanxiong Hort, Paeonia lactiflora Pall, Atractylodes macrocephala, Alisma Plantago-aquatica Linn, and Poria cocos, provided by the First Affiliated Hospital of Guangzhou University of Chinese Medicine. The six raw herbs were mixed in the ratio of
3:16:8:4:8:4, decocted in water twice, and the filtrates were mixed into a final concentration of $1.5 \mathrm{~g} / \mathrm{mL}$. Nimodipine was purchased from Bayer Healthcare Limited (batch number: H20003010).

\section{Animals and treatment}

In accordance with policies and procedures described in the Guidelines for the Care and Use of Laboratory Animals published by the National Research Council, the present experiments involving animals was approved by Guangzhou University of Chinese Medicine Animal Ethics Committee. Adult female Sprague-Dawley rats (weighing 250-300 g, Guangdong Provincial Institute of Traditional Chinese Medicine, Guangzhou, China) were used in the research. The rats were all maintained and housed in a specific pathogen-free animal room at $22^{\circ} \mathrm{C} \pm 2{ }^{\circ} \mathrm{C}$ with automatic light cycles (12 hours light/dark) and a relative humidity of 40\%-60\%. Food and tap water were offered and libitum throughout the study. BCCAO was carried out in the rats according to a previous study. ${ }^{26}$ In brief, animals were anesthetized using $1 \%$ pentobarbital sodium $(50 \mathrm{mg} / \mathrm{kg}$, i.m.) and the two common carotid arteries of rats were bluntly separated and permanently occluded with a silk suture. The animals in the sham group underwent identical procedures, except the common carotid arteries that were simply exposed without occlusion.

Six-month-old rats were divided into five groups at random: sham group $(n=12)$, model group $(n=12)$, low-dose DSS group $[1.8 \mathrm{~g} /(\mathrm{kg} \cdot \mathrm{d}), n=12]$, high-dose DSS group $[7.2 \mathrm{~g} /(\mathrm{kg} \cdot \mathrm{d}), n=12]$, and Nimodipine group [20 $\mathrm{mg} /(\mathrm{kg} \cdot \mathrm{d})$, $n=12]$. Rats were treated with distilled water, DSS, and Nimodipine, respectively, once per day for 4 weeks.

\section{Reagents}

The reagents were as follows: Rabbit anti-p-IKK (1:2000, ab194519; Abcam), rabbit anti-IKK (1:2000, ab32135; Abcam), rabbit anti-p-I $\kappa \mathrm{B}$ (1:1000, ab32518; Abcam), rabbit anti-I $\kappa \mathrm{B}(1: 1000,2859 \mathrm{~S}$; Cell Signaling Technology), rabbit anti-p-NF- $\kappa$ B (1:1000, \#3033; Cell Signaling Technology), rabbit anti-NF- $\kappa$ B (1:1000, \#8242; Cell Signaling Technology), rabbit anti-Bcl-2 (1:500, \#2876; Cell Signaling Technology), rabbit anti-Bax (1:500, \#2772; Cell Signaling Technology), rabbit anti-Caspase-3 (1:500, \#9662; Cell Signaling Technology), rabbit anti-LRP1 (1:4000, ab92544; Abcam), rabbit anti-RAGE (1:1000, ab3611;

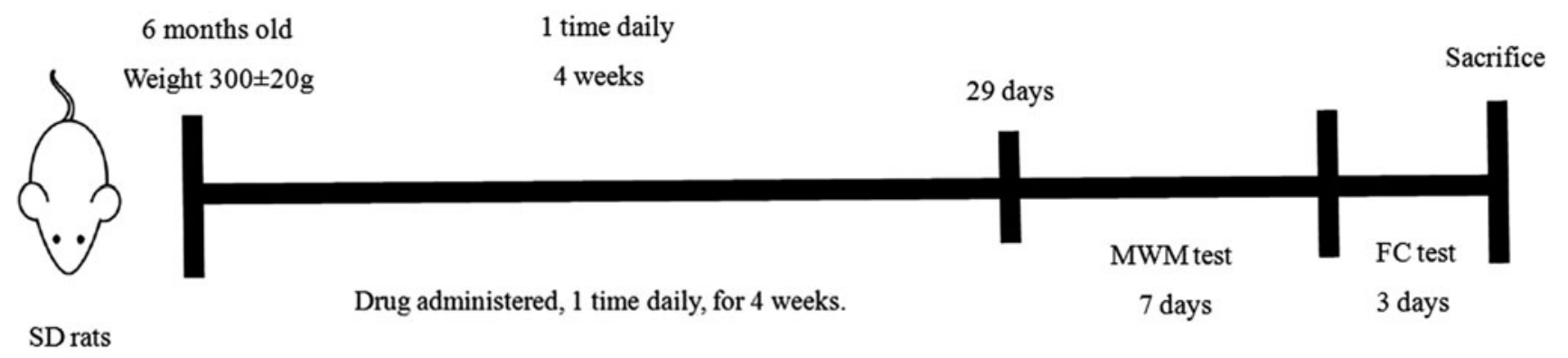

FIG. 1. Scheme of the experimental design. This is a schematic of the present experiment. FC, fear conditioning; MWM, Morris water maze; SD, Sprague-Dawley. 
Abcam), and mouse anti- $\beta$-actin (1:60,000, A5441; Sigma); anti-rabbit IgG, horseradish peroxidase (HRP)-linked antibody (1:1000, \#7074; Cell Signaling Technology); antimouse IgG, HRP-linked antibody (1:1000, \#7076; Cell Signaling Technology); malondialdehyde (MDA, Lot: A003-01; Nanjing Jiancheng Bioengineering Institute), superoxide dismutase (SOD, Lot: A001-01; Nanjing Jiancheng Bioengineering Institute), reactive oxygen species (ROS, Lot: E004; Nanjing Jiancheng Bioengineering Institute); enzyme-linked immunosorbent assay (ELISA) kits: rats amyloid beta peptide 1-42 was purchased from the Wuhan Huamei Bioengineering Institute (Wuhan, China; Lot: R09014760). Rats tumor necrosis factor $\alpha(\mathrm{TNF}-\alpha)$ Emax immunoassay kit $(6.25$ pg/mL/400 pg/mL, Lot: Z01019334) and rats IL- $1 \beta$ Emax immunoassay kit $(62.5 \mathrm{pg} / \mathrm{mL} / 4000$ pg/mL, Lot: X24019335) were from CUSABIO.

\section{Morris water maze test}

Morris water maze test was carried out based on the method of Morris. ${ }^{27}$ The pool was divided into four imaginary quadrants spatially. In the target quadrant of the pool, $2 \mathrm{~cm}$ below the water surface, a circular and transparent escape platform $(10 \mathrm{~cm}$ diameter $)$ was placed. We performed the visible platform test (water in the pool is clear and transparent) on the first training day before the hidden platform test. Rats were gently placed into the pool, with drop location changing for each trial randomly. Once the rat reached the platform, the escape latency was recorded. The rats were guided to the platform by the trainer and to remain on the platform for 20 seconds; if they failed to reach the platform within 60 seconds, the escape latency was recorded as 60 seconds. After visible platform test, we dyed the pool black, rats were given a position navigation test (hidden platform test) for 5 days. On the sixth day, we removed the platform, rats were permitted to explore in the pool for 60 seconds. Crossing frequency through the platform position and time spent in the target quadrant that indicated the degree of memory consolidation were measured.

\section{Fear conditioning test}

Fear conditioning test (FCT) was carried out as described in a previous study. ${ }^{28}$ In the first part of the test, each rat was allowed to have free activities in the test box for $300 \mathrm{sec}-$ onds. The presentation of a $2-\mathrm{Hz}$ pulsating tone $(80 \mathrm{~dB}$, $3000 \mathrm{~Hz}$ ) was persisted for 28 seconds. The tone was followed by a mild foot shock ( $0.8 \mathrm{~mA}$ for 2 seconds) immediately. There were six cycles and each cycle was spaced 20 seconds apart. Each rat was permitted to stay in the chamber for a total of 300 seconds. The second part of test was carried out at 3 hours and at 24 hours after the first part, respectively. In the second part, the mild foot shock $(0.8 \mathrm{~mA}$ for 2 seconds) was removed. By measuring the duration time that the rat demonstrated "freezing behavior," we could assess the function of associative memory in rats.

\section{Western blot}

The tissues were homogenized and lysed in sample buffer (5\% saline solution). The lysate was centrifuged at $12,000 \mathrm{~g}$ for 10 minutes at $4^{\circ} \mathrm{C}$, then denatured by boiling at $100^{\circ} \mathrm{C}$ with 1:4 loading buffer. The same amount of protein $(30 \mu \mathrm{g})$ was fractionated by sodium dodecyl sulfate-polyacrylamide gel electrophoresis (SDS-PAGE), subsequently transferred onto the PVDF membranes. The membranes were soaked in $5 \%$ skim milk that dissolved in TBST for 1 hour at room temperature, and then incubated with a primary antibody overnight at $4^{\circ} \mathrm{C}$. After washing with TBST, the membranes were incubated with appropriate secondary antibodies coupled to HRP for 1 hour at room temperature. Immunoreactive bands were visualized with an enhanced chemiluminescence kit (Pierce Biotechnology, Rockford, IL) and Chem Doc XRS with Quantity One software (Bio-Rad, Hercules, CA). The bands were scanned, and intensities of the bands were measured using Image-Pro Plus 6.0 analysis software.

\section{Enzyme-linked immunosorbent assay}

TNF- $\alpha$ and IL- $1 \beta$ in cortex homogenates and A $\beta 1-42$ were quantified using ELISA kits. The tissues were dissociated using $2 \%$ sodium dodecyl sulfate. Then, the homogenates were centrifuged at $14,000 \mathrm{rpm}$ for 30 minutes at $4^{\circ} \mathrm{C}$. The supernatant fractions were collected to detect the levels of TNF- $\alpha$, IL- $1 \beta$, and SDS-soluble A $\beta 1-42$. Samples were compared with the standard curves to calculate the levels of TNF- $\alpha$, IL- $1 \beta$, and SDS-soluble A $\beta 1-42$ in extracts.

\section{Measurement of MDA, ROS, and SOD}

The total protein concentration of the supernatant was determined using a bicinchoninic acid protein assay. Then the supernatant was used to detect the levels of MDA, ROS, and SOD in cortex according to the manufacturer's instructions.

\section{Nissl's staining}

Brain slices were washed in xylene and then rehydrated through ethanol and double-distilled water. The sections were soaked in Nissl's dye liquor for 5 minutes at room temperature. Double-distilled water was used to rinse the slices and then the slices were dehydrated through alcohol, cleared in xylene. A light microscope and LEICA QWin Plus were used to analyze slice images.

\section{Statistical analysis}

SPSS 19.0 software (SPSS, Inc., Chicago, IL) was used to analysis all experimental values given as mean \pm standard error of the mean. One-way analysis of variance was used to analyze differences in data among the groups, which was followed by Dunnett's significant post hoc test for pairwise multiple comparisons. Student-Newman-Keuls test was used when there was homogeneity of variance, and the rank sum test was used when the variance was not uniform. $p<0.05$ was considered to indicate a statistically significant difference.

\section{Results \\ DSS improved learning and memory performance in $\mathrm{VCl}$ rats}

In this study, we used Morris water maze and FCTs to assess the impacts of DSS on learning and memory ability in VCI rats.

In the Morris water maze test, we compared the escape latency of the rats in five experimental groups. We performed 
the visible platform test on the first training day before the hidden platform test and the escape latencies of the rats in five experimental groups did not show significant change (Fig. 2A) $(p>0.05)$. The escape latency in the model group was significantly elevated compared with the sham group in the positioning navigation test (Fig. $2 \mathrm{~B}, \mathrm{C})(p<0.01$ vs. sham) and the escape latency of DSS high-dose and Nimodipine treatment groups performed shorter compared with the model group (Fig. 2B, C) $(p<0.01$ and $p<0.05$, respectively vs. model). The probe test showed that crossing times and the swimming distance significantly differed among the five experimental groups (Fig. 2D, E). Compared with the model
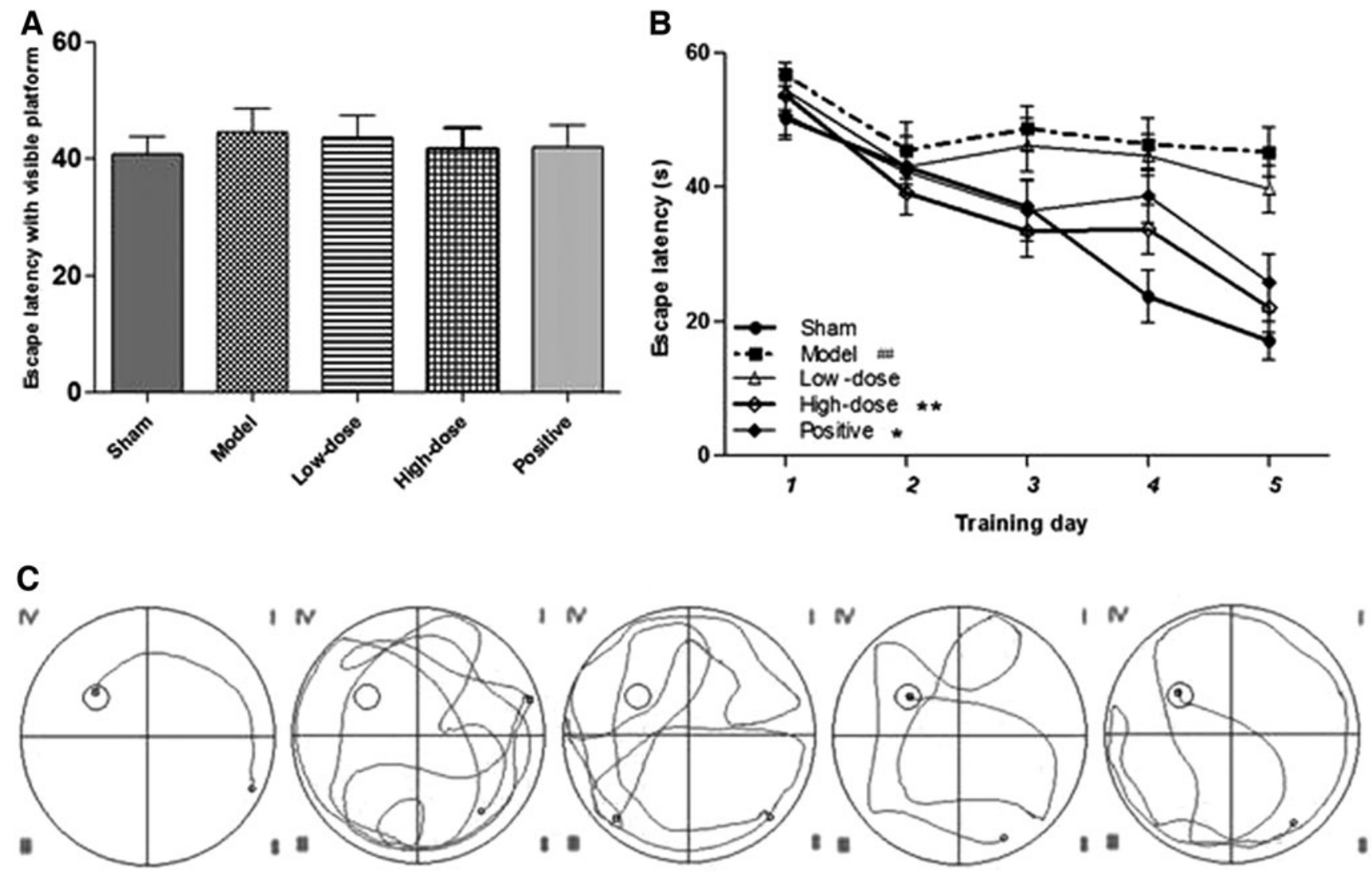

D

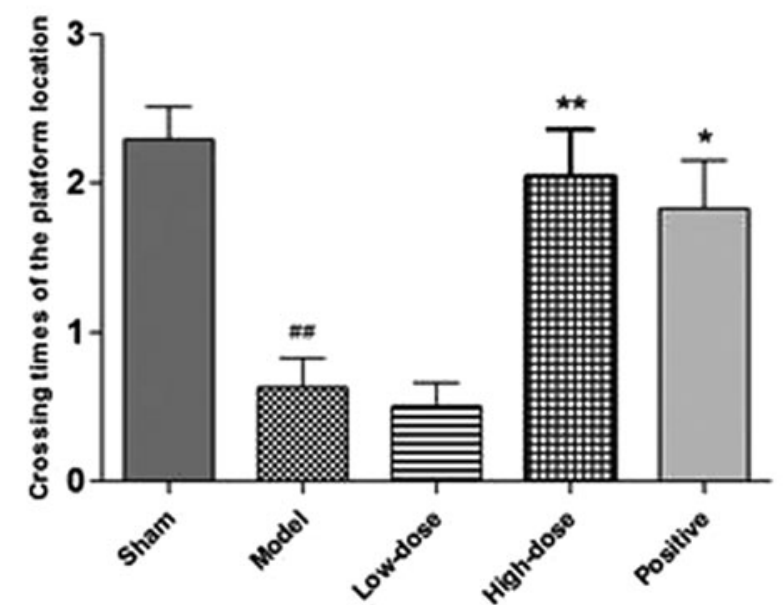

E

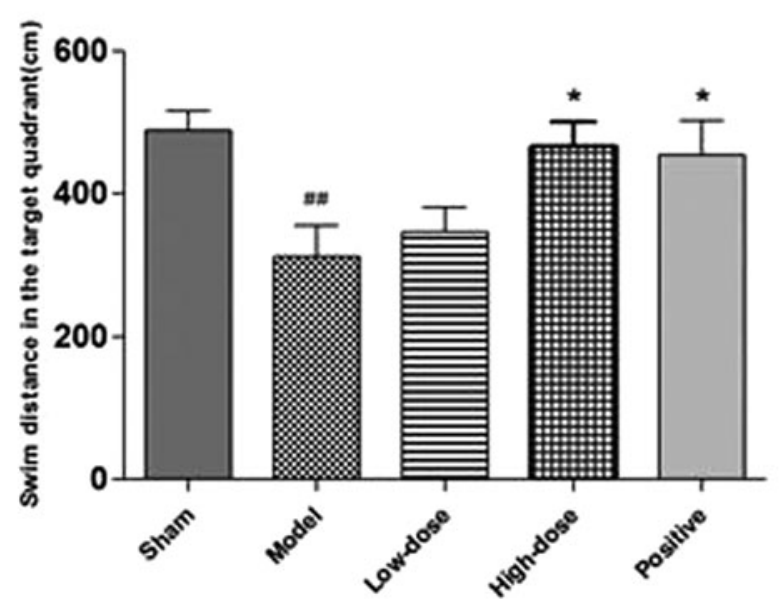

FIG. 2. DSS ameliorated cognitive impairment of VCI rats in Morris water maze test. (A) Escape latencies in water maze of the visible platform test. (B) Escape latencies during the five consecutive days positioning navigation test in Morris water maze. (C) Representative tracings of the animal's path. (D) Crossing times through the platform location in the probe trial. (E) Swimming distance in the target quadrant in the probe trial. Data are given as mean \pm SEM ( $n=12$ rats per group). ${ }^{\# \#} p<0.01$ versus the sham group. ${ }^{*} p<0.05$ and $* * p<0.01$ versus the model group. DSS, Danggui-Shaoyao San; SEM, standard error of the mean; VCI, vascular cognitive impairment. 
group, the DSS high-dose and Nimodipine groups significantly crossed more times (Fig. 2D) $(p<0.01$ and $p<0.05$, respectively vs. model). DSS high-dose and Nimodipine groups also significantly swam longer distance in the target quadrant compared with the model group (Fig. $2 \mathrm{E})(p<0.05$ and $p<0.05$, respectively vs. model).

In the FCT, the 3-hour test demonstrated that there were no significant discrepancies in freezing time between any two of the experimental groups (Fig. 3A). However, as the 24 hours test demonstrated, the freezing time of the sham group was significantly longer compared with the model group and the freezing time of the DSS high-dose group was significantly longer compared with the model group either (Fig. 3B) $(p<0.05$ vs. model).

The results of two behavior tests revealed that DSS improved the learning and memory ability in VCI rats.

\section{DSS treatment did not affect $A \beta 1-42$ levels through elevating $L R P 1$ expression in $\mathrm{VCl}$ rats}

Consistent with the findings of a previous study, LRP1 plays a critical role in brain $\mathrm{A} \beta$ clearance and neuroinflammation regulation in neurons and vascular mural cells. LRP1 also plays an important role in Abeta clearance in astrocytes, pericytes, and endothelial cells as shown before. $^{29-32}$ In these experiments, remarkably decreased expression of LRP1 was observed in the cortex and hippocampus of VCI rats, whereas after treatment with DSS or Nimodipine, the proteins restored to the normal levels (Fig. 4A-D) ( $p<0.01$ and $p<0.05$, respectively vs. model). Next, we measured the protein levels of the receptor for advanced glycation end products (RAGE), but there was no significant discrepancy between the groups (Fig. 4A-D). To uncover the deep mechanism of DSS in mitigating cognition deficiency in VCI rats, we next measured the levels of $\mathrm{A} \beta 1-$ 42. It was elevated in the hippocampus of model group compared with sham group (Fig. 4E) ( $p<0.05$ vs. sham). However, the levels of $\mathrm{A} \beta 1-42$ had no significant change in treatment groups compared with model group. These results demonstrate that the elevated levels of LRP1 did not have a significant effect on SDS-soluble A $\beta 1-42$ levels by DSS treatment in VCI rats.

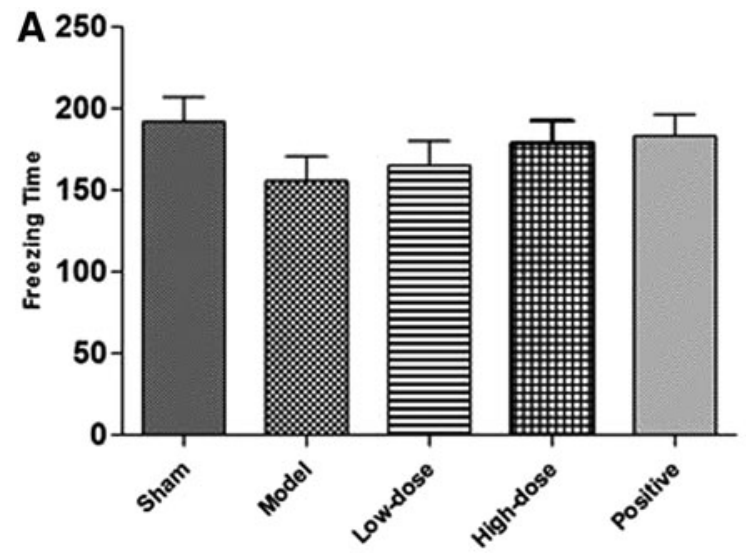

\section{DSS diminished proinflammatory cytokine production in $\mathrm{VCl}$ rats}

In addition to the role as an endocytic receptor, LRP1 also functions in cell signaling, regulates gene transcription, and inhibits inflammation. ${ }^{33,34}$ To determine whether LRP1 regulates expression of neuroinflammation, we first determined the levels of proinflammatory cytokine in VCI rats. The expression of TNF- $\alpha$ and IL- $1 \beta$ significantly increased both in the hippocampus of VCI rats (Fig. 5A, B) $(p<0.01$ and $p<0.01$, respectively vs. sham). The levels of TNF- $\alpha$ and IL- $1 \beta$ in DSS high-dose and Nimodipine groups decreased (Fig. 5A, B) $(p<0.01$ and $p<0.01, p<0.05$ and $p<0.05$, respectively vs. model). In response to chronic cerebral hypoperfusion (CCH), TNF- $\alpha$ and IL- $1 \beta$, which are proinflammatory cytokines, were significantly elevated in the hippocampus. The elevated levels of proinflammatory cytokines in VCI rats clearly emerged with a significant increment already in early pathological process of VCI. These features also showed that high levels of LRP1 might inhibit the expression of TNF- $\alpha$ and $1 \mathrm{~L}-1 \beta$ in VCI rats after treatment with DSS. In addition, a previous study showed that membrane-anchored LRP1 plays as a suppressor of inflammation by downregulating cell surface TNF- $\alpha$ and cell signaling through the IKK/NF- $\kappa \mathrm{B}$ pathway. ${ }^{35}$

\section{$D S S$ regulated IKK/NF- $\mathrm{B}$ pathway by regulating $L R P 1$ in $\mathrm{VCl}$ rats}

$\mathrm{NF}-\kappa \mathrm{B}$ functions as a major regulator of inflammation, cell survival, and as a mediator of the response to TNF- $\alpha{ }^{36}$ To identify candidate signaling cascades involved in the regulation of neuroinflammation expression by LRP1, we examined protein expression of p-IKK, IKK, p-I $\kappa \mathrm{B}, \mathrm{I} \kappa \mathrm{B}$, $\mathrm{p}-\mathrm{NF}-\kappa \mathrm{B}$, and NF- $\kappa \mathrm{B}$. In response to TNF- $\alpha$, phosphorylated IKK was substantially increased in hippocampus of model group compared with sham group (Fig. 6A, B) ( $p<0.01$ vs. sham) and decreased after the administration of DSS high-dose (Fig. 6A, B) $(p<0.01$ vs. model). Meanwhile, there was no significant change on IKK (Fig. 6A, B). Moreover, there was an increment in the active form of the phosphorylated $\mathrm{I} \kappa \mathrm{B}$ in the hippocampus of model group compared with sham group (Fig. 6A, C) $(p<0.01$ vs. sham),

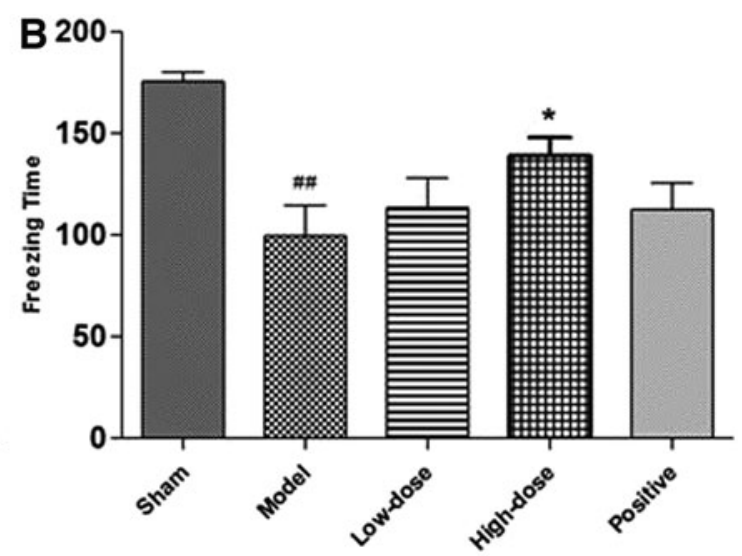

FIG. 3. DSS ameliorated cognitive impairment of VCI rats in FCT. (A) Freezing time of 3 hours after anesthesia in FCT. (B) Freezing time of 24 hours after anesthesia in FCT. Data are given as mean \pm SEM $\left(n=12\right.$ rats per group). ${ }^{\# \#} p<0.01$ versus the sham group. ${ }^{*} p<0.05$ versus the model group. FCT, fear conditioning test. 

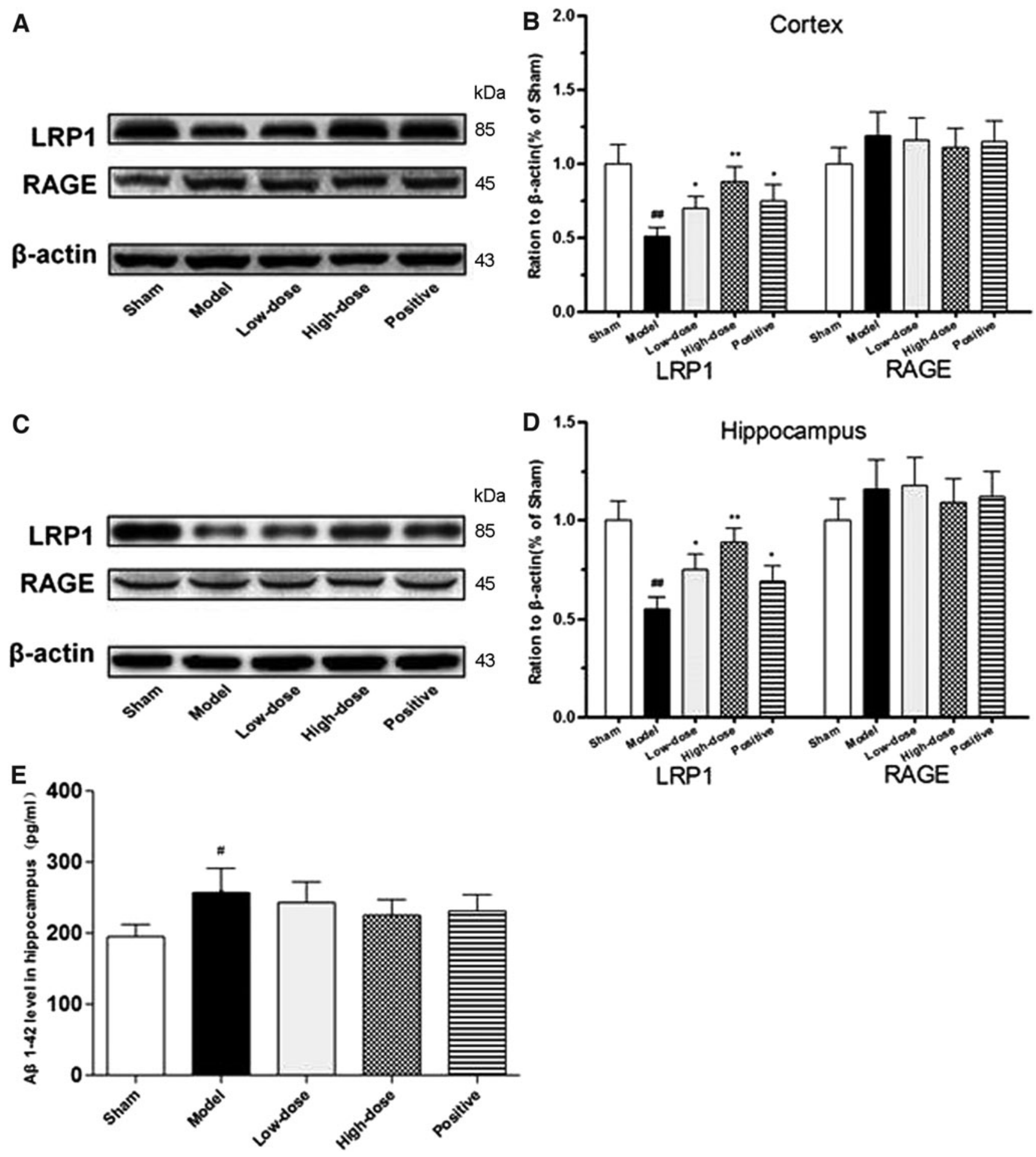

FIG. 4. DSS restored the levels of LRP1 in VCI rats. (A-D) LRP1 and RAGE protein levels in the cortex and hippocampus after DSS treatment are determined by western blot analysis. (E) ELISA results of A $\beta 1-42$ levels in the VCI rats after treatment of DSS. Data are given as mean \pm SEM $\left(n=12\right.$ rats per group). ${ }^{\#} p<0.05$ and ${ }^{\# \#} p<0.01$ versus the sham group. ${ }^{*} p<0.05$ and ${ }^{* *} p<0.01$ versus the model group. ELISA, enzyme-linked immunosorbent assay; LRP1, low-density lipoprotein receptor-related protein 1; RAGE, receptor for advanced glycation end products.

and decreased after the treatment of DSS high-dose group (Fig. 6A, C) $(p<0.01$ vs. model). The total $\mathrm{I} \kappa \mathrm{B}$ in the hippocampus of VCI rats also decreased after the treatment of DSS high-dose group (Fig. 6A, C) $(p<0.01$ vs. model). To further evaluate the consequence of IKK/NF- $\kappa \mathrm{B}$ path- way, we next measured the expression levels of phosphorylated NF- $\kappa \mathrm{B}$ and NF- $\kappa \mathrm{B}$. The phosphorylated NF- $\kappa \mathrm{B}$ in VCI rats was elevated compared with sham group (Fig. 6A, D) $(p<0.01$ vs. sham $)$, but sharply decreased after the treatment of DSS high dose (Fig. 6A, D) $(p<0.01$ vs. model). 

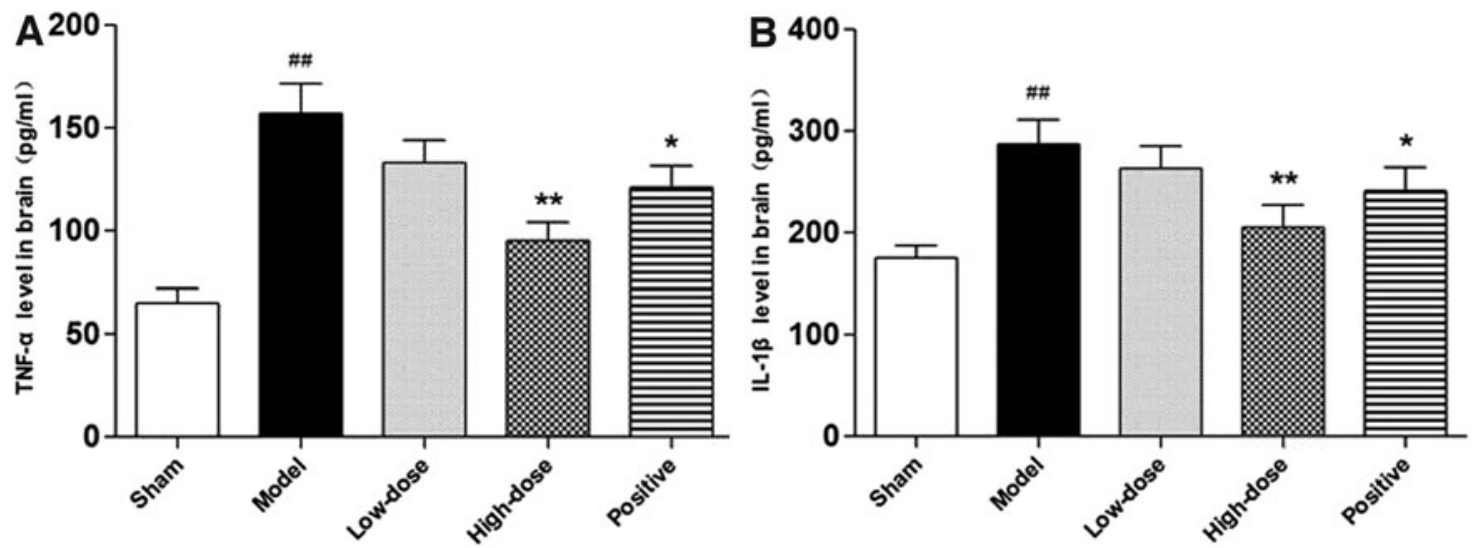

FIG. 5. DSS inhibited the production of proinflammatory cytokine in VCI rats. (A) ELISA results of TNF- $\alpha$ levels in the hippocampus. (B) ELISA results of interleukin-1 $\beta$ (IL-1 $\beta$ ) levels in the hippocampus. Data are given as mean \pm SEM $(n=12$ rats per group). ${ }^{\# \#} p<0.01$ versus the sham group. ${ }^{*} p<0.05$ and $* * p<0.01$ versus the model group. TNF- $\alpha$, tumor necrosis factor $\alpha$.

These results indicated that IKK-induced phosphorylation of $\mathrm{I} \kappa \mathrm{B}$ promotes $\mathrm{I} \kappa \mathrm{B}$ degradation and release of activated NF$\kappa \mathrm{B} .{ }^{37,38}$ Our research predicting the effect of DSS on neuroprotection might be related to LRP1 by inhibiting the activation of IKK/NF- $\kappa \mathrm{B}$ signal pathway.

\section{DSS ameliorated neuronal apoptosis in $\mathrm{VCI}$ rats}

Based on a previous study, we hypothesized that the brain is in low blood flow perfusion for a long period, which can cause cerebral ischemia, hypoxia, and neuroinflammation, resulting in the neuronal apoptosis and oxidative stress in the cerebrum, eventually leading to VCI. To test this hypothesis, we measured the active forms of the effectors of neuronal apoptosis, including $\mathrm{Bcl}-2$, Bax, and cleaved Caspase- 3 in VCI rats. The ratio of Bcl-2/Bax decreased and cleaved Caspase-3 increased in VCI rats in hippocampus (Fig. 7A-D) $(p<0.01$ and $p<0.01$, respectively vs. sham). The ratio of $\mathrm{Bcl}-2 / \mathrm{Bax}$ increased and the levels of cleaved Caspase-3 decreased in hippocampus after treatment of DSS (Fig. 7A-D) $(p<0.01$ and $p<0.01$, respectively vs. model). As one marker of neurodegeneration in the hippocampus and the neural characteristic structures, the Nissl bodies can reflect the neuron survival state. Nissl's staining showed that neurons were diffusely deteriorated, a great number of Nissl bodies lost in the CA1 subfield of the hippocampal formation in VCI rats (Fig. 7E, F) ( $p<0.01$ vs. sham). In contrast, the neurons in the CA1 area in DSS high-dose group showed less neuronal damage, deeply stained, normal form neurons, and the morphology was well preserved (Fig. 7E, F) $(p<0.05$ vs. model). These results demonstrated that DSS ameliorates neuronal apoptosis and play to the advantage of neuroprotection in VCI rats.

\section{DSS alleviated the oxidative stress in $\mathrm{VCI}$ rats}

This study found MDA and ROS activity also increased in VCI rats (Fig. 8A, B) $(p<0.01$ and $p<0.01$, respectively vs. sham). After being treated by DSS, the levels of MDA and ROS restored to normal levels (Fig. 8A, B) $(p<0.01$ and $p<0.01$, respectively vs. model). To further evaluate the consequence of oxidative stress in VCI rats, we next measured the expression levels of SOD, which were sharply diminished in VCI rats (Fig. 8C) $(p<0.01$ vs. sham) and elevated after the treatment of DSS and Nimodipine (Fig. 8C) $(p<0.05$ and $p<0.05$, respectively vs. model). These results indicated that DSS could ameliorate oxidative stress in VCI rats. This might be one of the neuroprotection functions of DSS.

\section{Discussion}

In this research, we showed the neuroprotective effect of DSS against $\mathrm{CCH}$-induced cognitive impairment in VCI rats. We also found an elevation of LRP1 activation in hippocampus and cortex of VCI rats. This suggested that the neuroprotective effects of DSS might in part be ascribed to enhancing LRP1 expression. We speculated that DSS might mediate IKK/NF- $\kappa \mathrm{B}$ pathway in VCI rats by regulating LRP1 expression. Our results indicate that DSS may be a useful therapeutic agent in $\mathrm{CCH}$-induced cognitive impairment (Fig. 9).

VCI is caused by a range of cardiovascular or cerebrovascular conditions that may lead to cerebral ischemic, hypoperfusion, or hemorrhagic brain lesions with the loss of cognitive functions. ${ }^{11,39}$ Considerable evidence indicates that $\mathrm{CCH}$ contributes to the development and progression of dementia through neuroinflammation, oxidative stress, and neuronal apoptosis, instead of the normal functionality of the recovery. ${ }^{40}$ Despite the involvement of different mechanisms in VCI, increasing evidence indicates that neuroinflammation is an important pathological feature and an early event in the pathogenesis of VCI. ${ }^{41}$ Emerging data indicated that excessive or uncontrolled inflammation leads to worse clinical outcome in acute cerebrovascular diseases. ${ }^{42,43}$

LRP1 is abundantly expressed in diverse tissues including brain, liver, and vasculature. A previous study revealed that LRP1 is involved in multiple pathways, including modulation of $\mathrm{A} \beta$ clearance, lipid transport, neuroprotection, and synaptic functions in dementia pathogenesis. ${ }^{4,45}$ LRP1 binds diverse extracellular ligands through cellular trafficking and/or signal pathways to recognize ApoE and $\mathrm{A} \beta$ in the pathogenesis of dementia. ${ }^{12}$ In addition, recent work by Yang et al. demonstrated that LRP1 suppresses microglial 
A
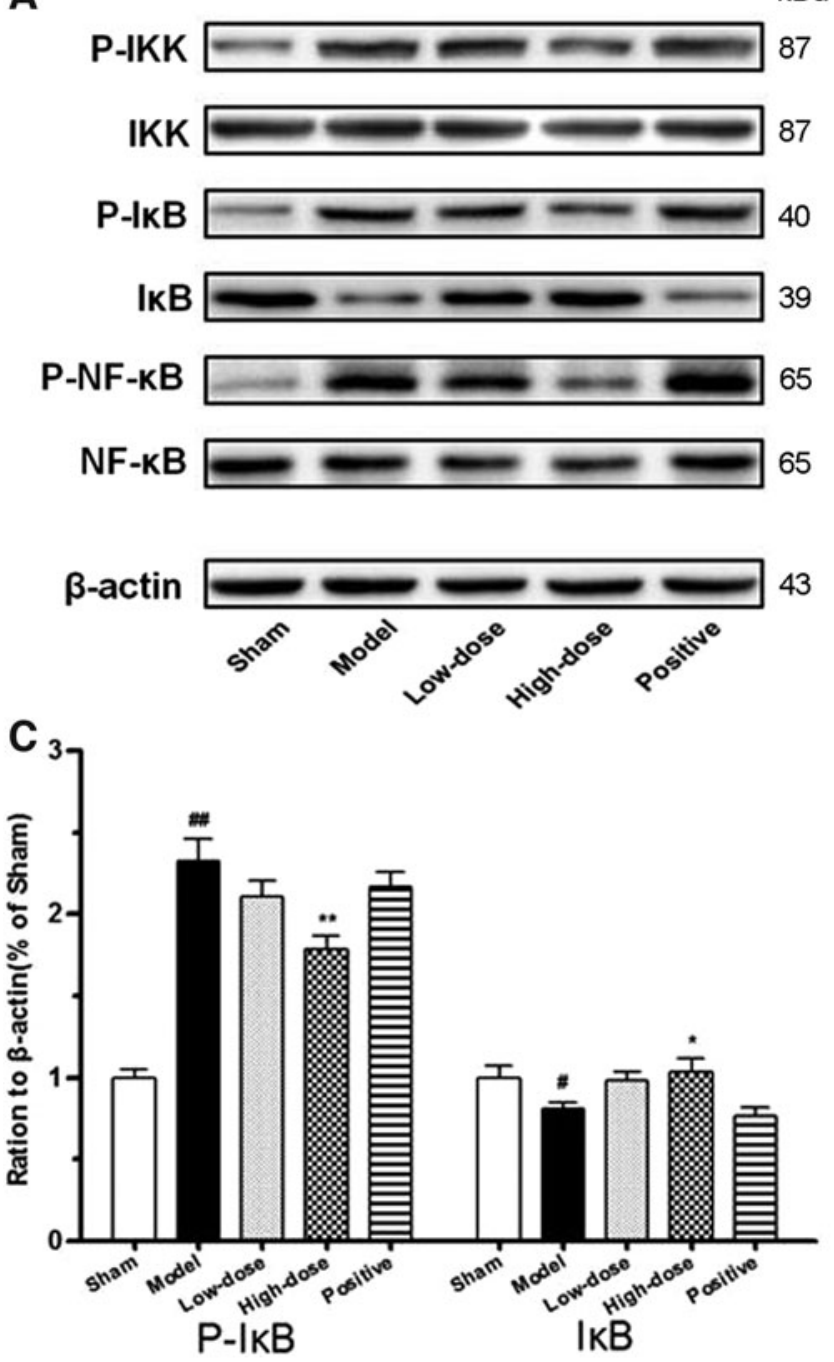

B
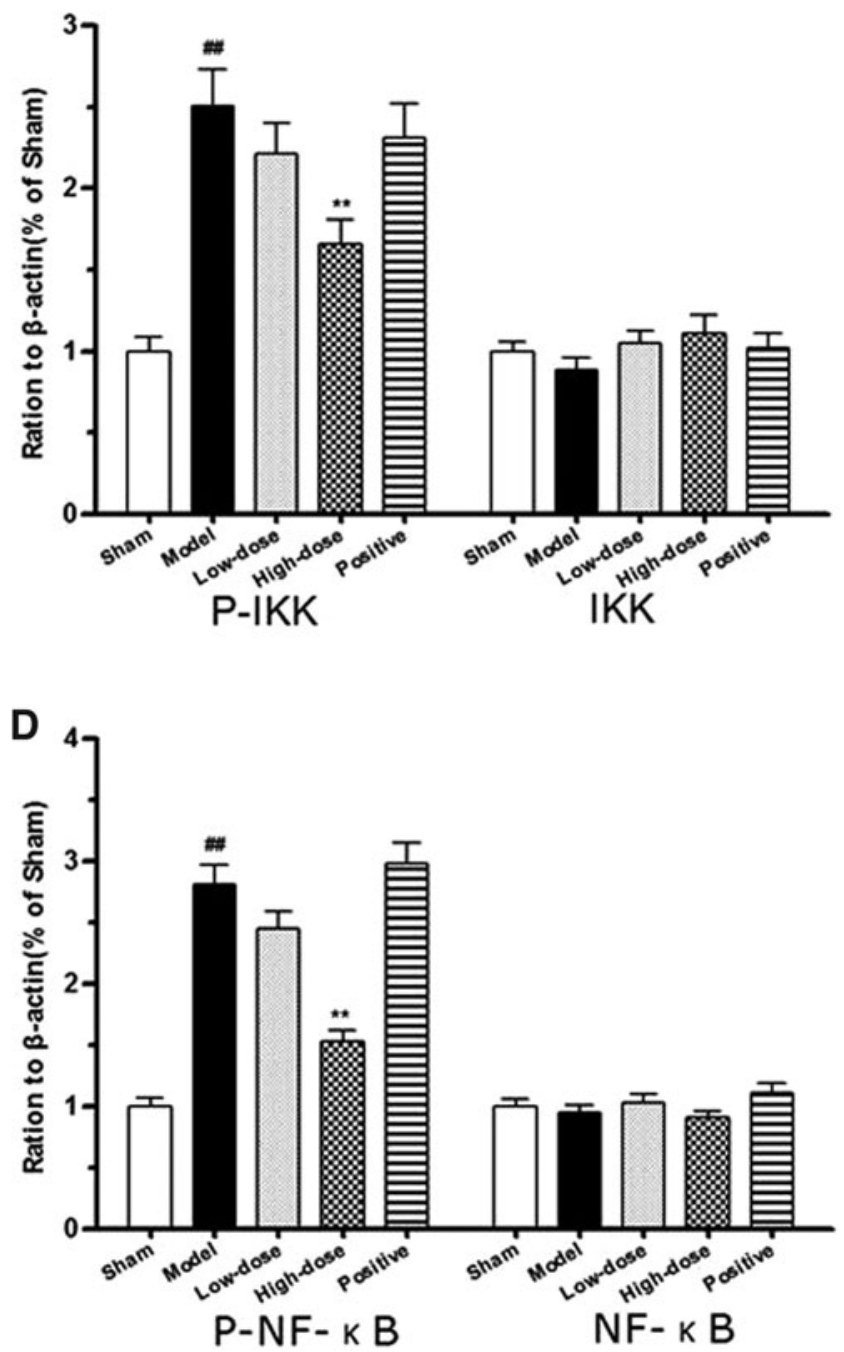

FIG. 6. DSS inhibited IKK/NF- $\kappa$ B pathway in VCI rats. (A) Western blot analysis of IKK/NF- $\kappa$ B signal pathway associated proteins in VCI rats after treatment of DSS. DSS restored the levels of phosphorylation-IKK, phosphorylation$\mathrm{I} \kappa \mathrm{B}$, and phosphorylation-NF- $\kappa \mathrm{B}$ proteins in VCI rats. Meanwhile, the levels of total $\mathrm{I} \kappa \mathrm{B}$ significantly decreased in the model group compared with sham group. (B-D) Quantification of the band intensities are presented in the adjacent graphs. Data are given as mean \pm SEM ( $n=12$ rats per group). ${ }^{\#} p<0.05$ and ${ }^{\# \#} p<0.01$ versus the sham group. ${ }^{*} p<0.05$ and ${ }^{* *} p<0.01$ versus the model group. IKK, I kappa B kinase; NF- $\kappa \mathrm{B}$, nuclear factor of kappa B.

activation by modulating c-Jun N-terminal kinase (JNK) and NF- $\kappa \mathrm{B}$ signal pathways, as a potential diseasemodifying target for the treatment of $\mathrm{AD} .{ }^{46}$ LRP1 is expressed by microglia, particularly at sites of inflammation, and may be involved in regulating microglial activation. Silencing LRP1 gene expression in microglia or treating microglia with receptor-associated protein activates NF- $\kappa \mathrm{B}$ and JNK and increases expression of inflammatory cytokines. ${ }^{46,47-49}$ In this study, we measured the $\mathrm{A} \beta$ transport protein in VCI rats, such as LRP1 and RAGE. As a result, the expression of LRP1 decreased and the levels of A $\beta 1-42$ were elevated in the model group compared with the sham group, whereas the expression of LRP1 increased after treatment of DSS. These results indicated that DSS elevated the levels of LRP1 in VCI rats.

Previous studies found that LRP1 in microglia directly regulates specific signal pathways critical for inflammatory responses, because downregulating LRP1 expression in microglia results in an increase of proinflammation cytokines. ${ }^{50}$ These reports suggest elevated LRP1 can inhibit the activity of the IKK/NF- $\kappa$ B pathway, as a system that may be modulated to either promote or inhibit macrophage inflammatory responses. ${ }^{51}$ This feature provides a mechanistic explanation for the anti-inflammatory activity of LRP1, which is evident in mouse models of atherosclerosis. ${ }^{52} \mathrm{We}$ hypothesize that under basal conditions, DSS could modulate neuroinflammation, neuronal apoptosis, and oxidative stress by targeting LRP1 in an autocrine pathway, which indirectly suppressed IKK/NF- $\kappa$ B pathway.

TNF- $\alpha$ is a potent proinflammatory cytokine that transduces the signals generated at TNF receptors to nuclear targets such as AP- 1 and NF- $\kappa \mathrm{B}$, which in turn induce genes involved in chronic and acute inflammatory responses. ${ }^{36,53}$ In response to TNF- $\alpha$, IL- $1 \beta$, and other agonists, the I $\kappa \mathrm{Bs}$ 


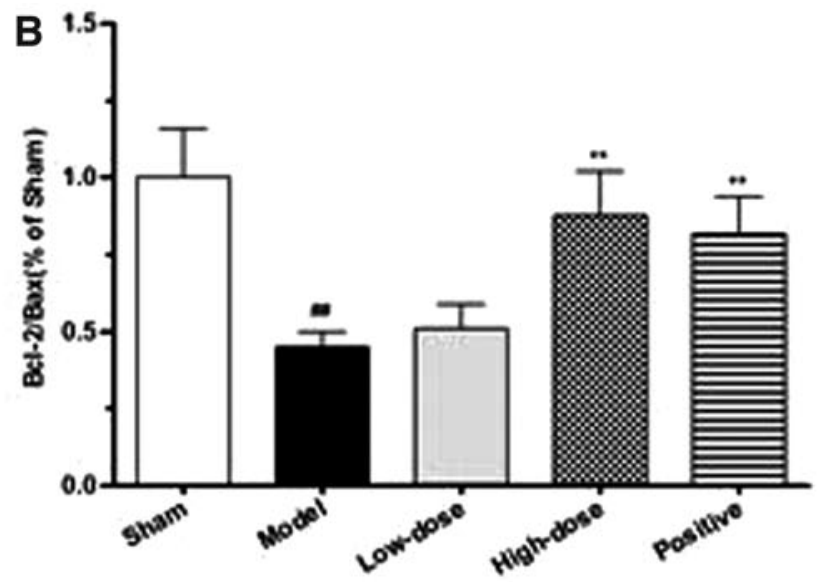

C
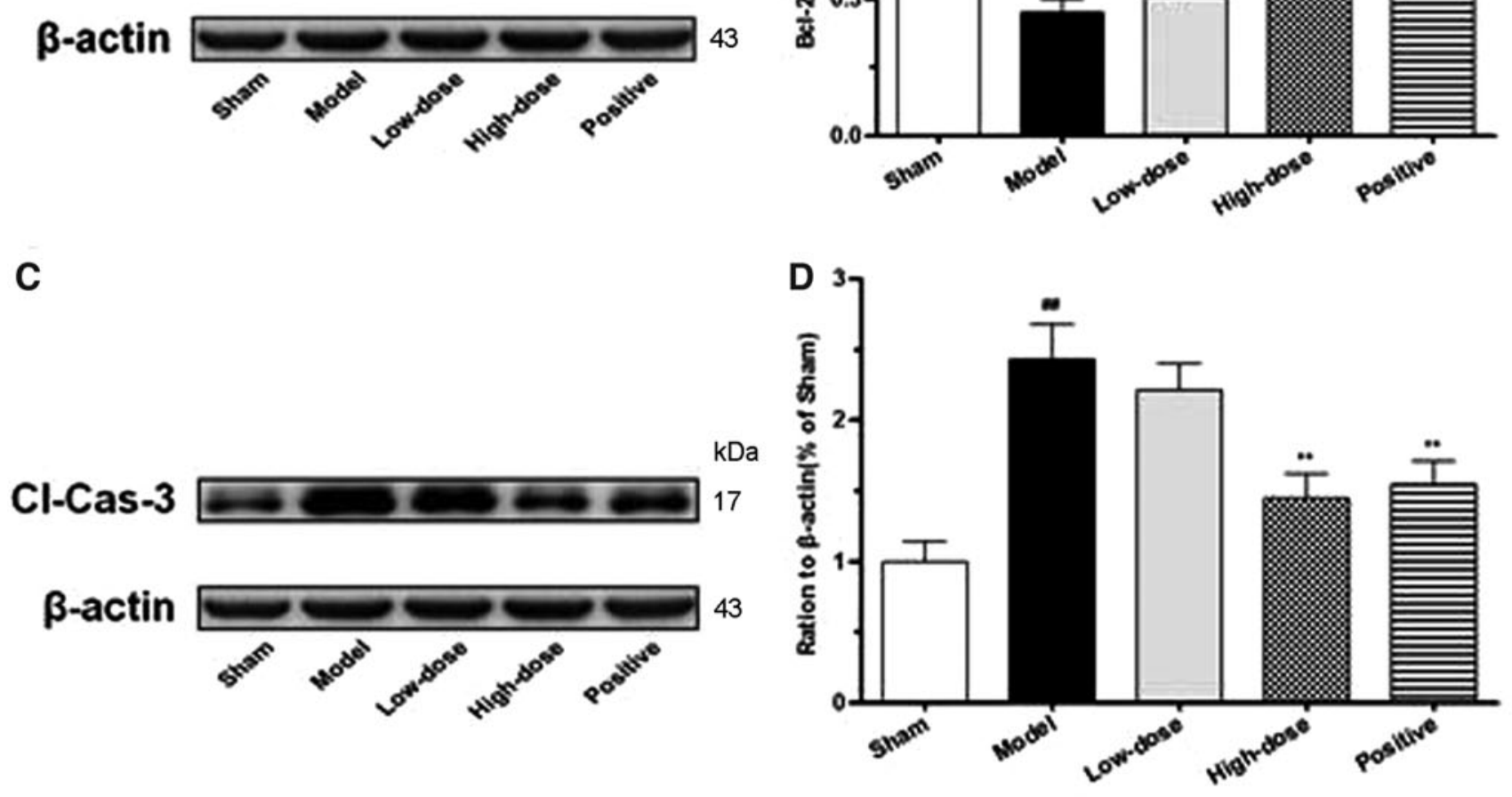

E
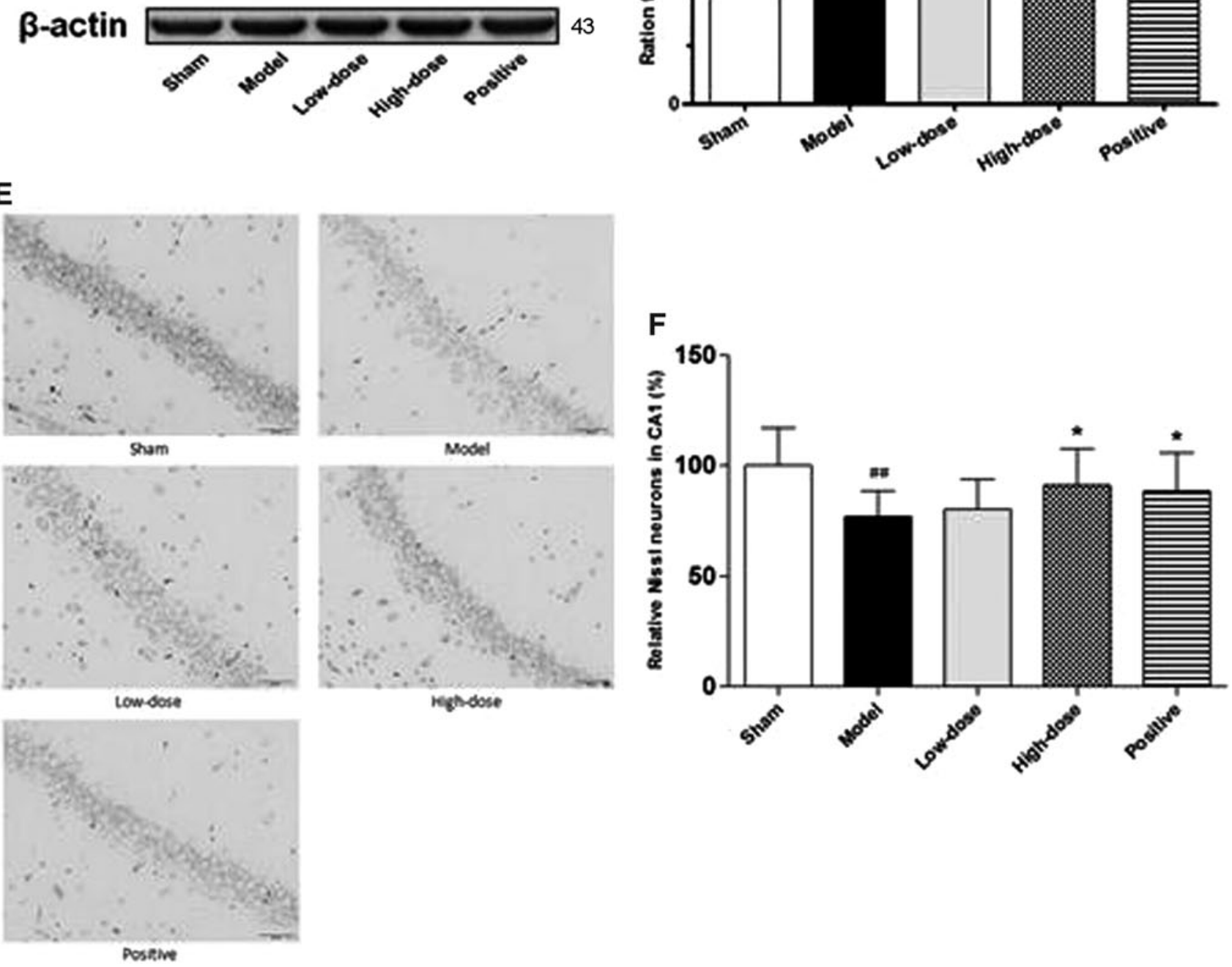

FIG. 7. DSS ameliorates neuronal apoptosis in VCI rats. (A, B) The ratio of Bcl-2 and Bax in the hippocampus. (C, D) The levels of cleaved Caspase-3 in the hippocampus. (E, F) The Nissl's staining in CA1 area of the hippocampus. Data are given as mean $\pm \operatorname{SEM}\left(n=12\right.$ rats per group). ${ }^{\#} p<0.05$ and ${ }^{\# \#} p<0.01$ versus the sham group. $* * p<0.01$ versus the model group. 

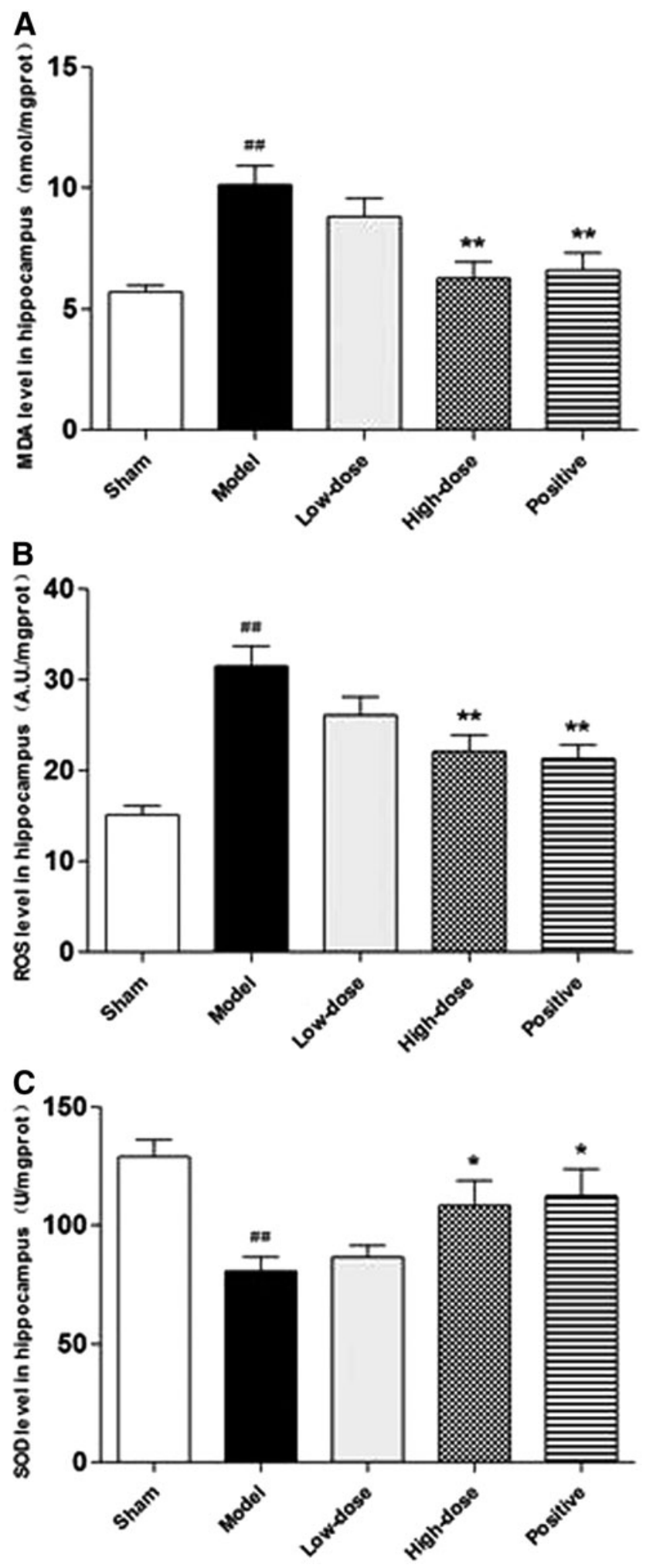

FIG. 8. DSS alleviated oxidative stress in VCI rats. (A-C) The levels of MDA, reactive oxygen (ROS), and SOD in the hippocampus. Note that MDA and ROS levels were selectively downregulated and SOD levels was elevated in the hippocampus after treatment of DSS. Data are given as mean \pm SEM $\left(n=12\right.$ rats per group). ${ }^{\# \#} p<0.01$ versus the sham group. $* p<0.05$ and $* * p<0.01$ versus the model group. MDA, malondialdehyde; ROS, reactive oxygen species; SOD, superoxide dismutase. are phosphorylated by the IKK complex, resulting in their ubiquitination, degradation, and nuclear translocation of the freed NF- $\kappa \mathrm{B}$. Once getting into the nucleus, NF- $\kappa \mathrm{B}$ transcriptional activity can be modulated further through phosphorylation by various protein kinases that are TNF- $\alpha$ and other proinflammation responsive. ${ }^{54}$ In APP/PS1 transgenic rats, the levels of proinflammation reduced astrocytosis, $\mathrm{A} \beta$ oligomerization, and deposition, and enhanced neurogenesis. ${ }^{55}$ Another study has shown that NF$\kappa \mathrm{B}$ and JNK pathways are both important to modulate the release of inflammatory cytokines and phagocytosis in AD pathogenesis. ${ }^{44}$ Numerous studies have demonstrated the activation of NF- $\kappa \mathrm{B}$ in astrocytes and that an astrocyte proinflammatory pathway may have important consequences for cognitive outcome in neurodegenerative disease. ${ }^{56}$ In this study, we found that high expression of TNF- $\alpha$ and IL- $1 \beta$ in VCI rats results in an increase of p-IKK, p-I $\kappa \mathrm{B}$ and a decrease of $\mathrm{I} \kappa \mathrm{B}$, meanwhile NF- $\kappa \mathrm{B}$ transcriptional activity is activated by $\mathrm{I} \kappa \mathrm{B}$ and various protein kinases. The importance of this process in controlling the activity of the IKK/NF- $\kappa \mathrm{B}$ pathway may be regulated transcription and expression of proinflammatory factor. After the treatment of DSS and Nimodipine, DSS could improve the neural function of VCI rats through the IKK/NF- $\kappa \mathrm{B}$ pathway. Actually, inflammatory factors emerging as key players in dementia increase the expression of cytokines (TNF- $\alpha$ and IL-1 $\beta$ ) and consequently, that of IKK/NF- $\kappa \mathrm{B}$ pathway. ${ }^{57,58}$ Nevertheless, emerging data indicate that the harmful stimulus is no longer present, blockade of inflammation is protective in common neurological diseases. ${ }^{59,60}$

Neuron apoptosis and oxidative stress are caused by a long time of cerebral ischemic, hypoperfusion, neuroinflammation, and hemorrhagic brain lesions with the decline of cognitive functions. ${ }^{61}$ Many studies have demonstrated that the suppression of apoptosis, which is mostly dependent on NF- $\kappa \mathrm{B}$, augments the inflammatory response to TNF- $\alpha$. Of importance, neuron apoptosis was mediated by various factors, such as Bcl-2, Bax, Caspases, A $\beta$, TNF- $\alpha$, ROS. ${ }^{62}$ The Bcl-2 protein family, including antiapoptotic protein Bcl-2 and proapoptotic protein Bax, plays a very important role in mitochondria-mediated apoptosis and is crucial to mitochondrial regulators during neuron apoptosis. ${ }^{63}$ A large number of studies indicated that overexpression of $\mathrm{Bcl}-2$ could inhibit the neuron apoptosis. ${ }^{64,65}$ Meanwhile, preclinical study found that the number of neuronal apoptosis in the hippocampus of adult rats was significantly reduced in knockout Bax gene. ${ }^{66}$ Many researches confirmed that Caspase-3 plays a critical role in neuron apoptosis and neuron cell remodeling, including the synaptic plasticity, cut of dendritic spines, and longtime history inhibition effect. $^{18-22}$ In addition, intense-activated Caspase-3 regulated to the proteolytic cleavage of polymeric tau protein in the ultimate apoptosis of cells in AD. ${ }^{67}$ Our work suggested that the expression of cleaved Caspase- 3 was increased in the brain tissues of the model group compared with sham group. Treatment of DSS high-dose and Nimodipine significantly reduced the expressions of cleaved Caspase- 3 and Bax protein, increased the protein expression of Bcl-2 and the ratio of $\mathrm{Bcl}-2 / \mathrm{Bax}$. Those results suggest that DSS can improve cognitive function and prevent neuronal injury in VCI rats by inhibiting neuronal apoptosis. In this study, neurons 


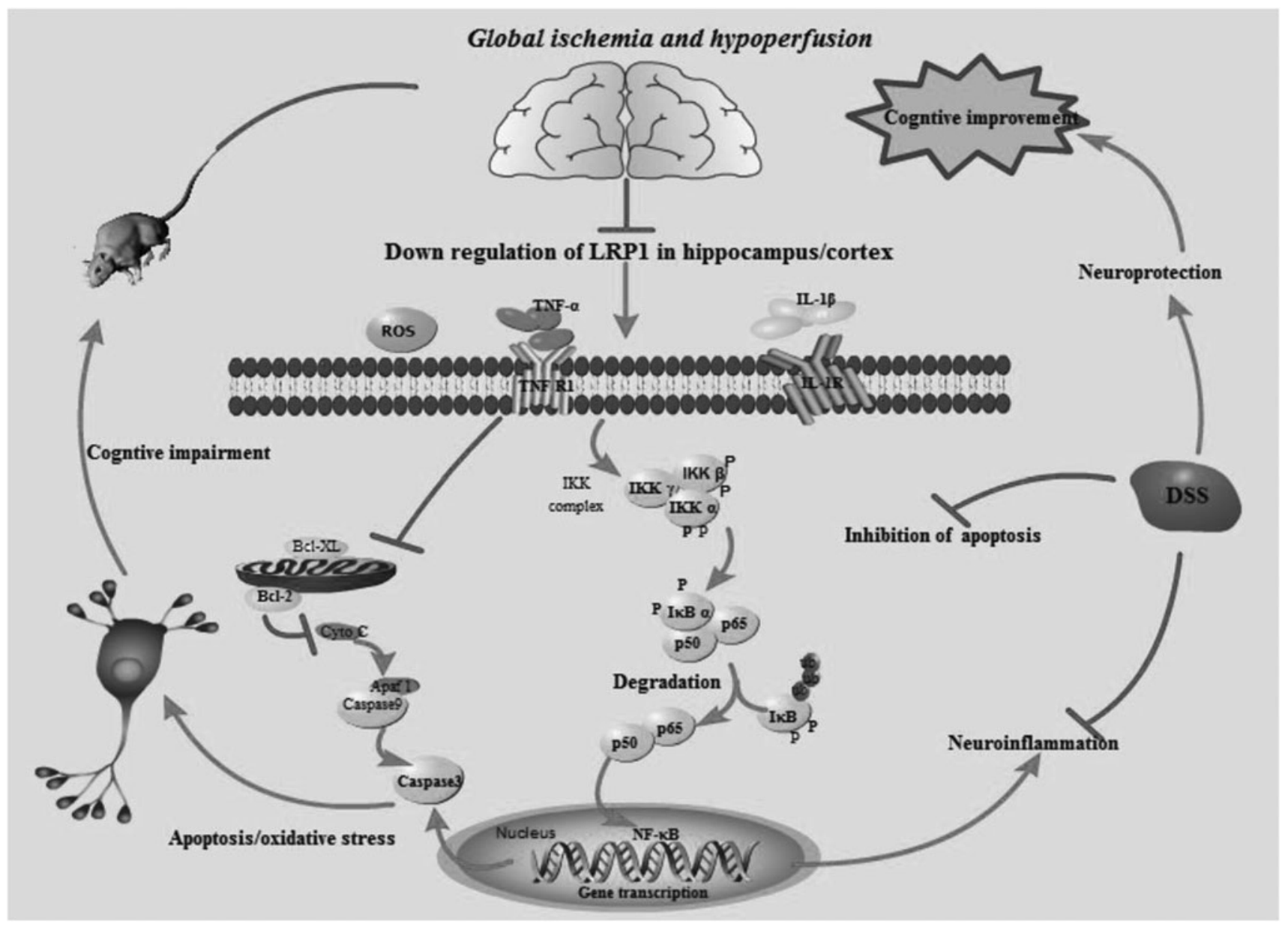

FIG. 9. Schematic diagram of LRP1-associated pathogenesis in VCI rats. An ischemia/hypoperfusion conditions leads to selective activation of proinflammatory mediators (such as IL- $1 \beta$, TNF- $\alpha$ ) in the hippocampus, CA1 neuron apoptosis, vascular damage, and a cognitive deficit. Under the hypoperfusion condition in the hippocampus, proinflammatory cytokine induces IKK/NF- $\kappa \mathrm{B}$ pathway, which in turn induce genes involved in chronic and acute inflammatory responses. Furthermore, neuron apoptosis and oxidative stress are mostly dependent on the IKK/NF- $\kappa \mathrm{B}$ pathway in response to the longtime of cerebral ischemic, hypoperfusion, neuroinflammation, and hemorrhagic brain lesions with the loss of cognitive functions. At the same time, DSS improves cognitive function and prevents neuroinflammation of VCI rats by targeting LRP1 led to an inhibiting effect on IKK/NF- $\kappa \mathrm{B}$ pathway.

were impaired owing to $\mathrm{CCH}$ but reversed by DSS. Meanwhile, the Nissl's staining also demonstrated the neuroprotective function of DSS.

The present study demonstrated that neuronal apoptosis plays a key role in the pathogenesis of VCI and DSS can significantly inhibit neuronal apoptosis. During the past decades, a vast number of studies provided evidences that increased levels of oxidative stress may lead to cerebrovascular changes. ${ }^{68}$ Based on our study, examining cells under basal conditions and in response to the oxidation reaction, we propose that MDA, ROS, and SOD may participate in this produce, among receptors that are indirectly regulated by $\mathrm{IKK} / \mathrm{NF}-\kappa \mathrm{B}$ pathway. ROS is a kind of molecules with oxidation ability mainly including the ultra oxygen anion, hydrogen peroxide, hydroxyl free radicals, singlet oxygen, and so on, which widely exists in the body and regulates the body's physiological and pathological process. ${ }^{69}$ Studies have shown that ROS can act as a signaling molecule to mediate cell signal transduction, thus inducing apoptosis. ${ }^{70}$ In addition, the effect of ROS-induced apoptosis may involve in increasing the ratio of Bax/ Bcl-2. ${ }^{71-73}$ After the cerebral ischemia injury, a large number of free radicals can be produced, damaging the cell membrane of neurons, resulting in lipid peroxidation and a large number of MDA. With the massive consumption of antioxidant enzymes, such as SOD, the body's ability to scavenge free radicals gradually decreases. ${ }^{74}$ Massive accumulation of free radicals direct damage to the brain, result in producing large numbers of free radicals, lipid peroxidation in the brain, causing the brain neurons, mitochondrial membrane injury and energy metabolism disorder, and finally lead to cell death. ${ }^{75}$ This feature demonstrated that the DSS protects against neuronal oxidative stress and improves the learning and memory ability in VCI rats. Based on our current findings, DSS might be a promising antiinflammatory and antioxidative stress agent for the treatment of VCI.

Taken together, our data clearly demonstrated that treatment with DSS could mitigate cognitive deficiency and inhibit the proinflammation factor. DSS inhibited the IKK/NF- $\kappa \mathrm{B}$ 
pathway by elevating levels of LRP1. DSS mitigated $\mathrm{CCH}$-associated cognitive decline and played a neuroprotective role in VCI rats that occur after carotid arteries occlusion. Thus, we propose that DSS can be used as a treatment to control cognitive impairment, inflammatory and oxidative process associated with VCI. Therefore, it might not only contribute to symptomatic improvement, but also play important roles for the treatment of neurodegenerative diseases by interfering with key factors of the disease. However, further mechanisms remain to be explored.

\section{Authors' Contributions}

The main article text and experiments were completed by H.C. and T.C. Figures 1 to 8 were all drawn by H.C. and Figure 9 was drawn by T.C. C.Y. and Y.W. prepared the Western blot and staining figures analysis. Q.Z., L.Z., and X.P. supported the behavioral tests and reviewed the describing article. H.Z. and L.L. checked the English grammar. Z.G., H.P., and Q.W. supervised the article and directed final version of all contents. All authors reviewed the final draft of the article and approved it for submission.

\section{Author Disclosure Statement}

No competing financial interests exist.

\section{Funding Information}

This work was supported by the National Natural Science Foundation of China (No. 81804004, No. 81673946, No. 81673627), China Postdoctoral Science Foundation (No. 2018M643207), Guangdong Provincial Major Science and Technology for Special Program (No. 2015A030302072), Shenzhen Municipal Health Commission Project (No. SZBC2018005), Shenzhen Science and Technology Project (No. JCYJ20160428174825490), and Shenzhen Medicine and Hygiene Sanming Project (No. SZSM201612081).

\section{References}

1. Hurd MD, Martorell P, Delavande A, et al. Monetary costs of dementia in the United States. N Engl J Med 2013;368: 1326-1334.

2. Choi BR, Lee SR, Han JS, et al. Synergistic memory impairment through the interaction of chronic cerebral hypoperfusion and amlyloid toxicity in a rat model. Stroke 2011; 42:2595-2604.

3. Casserly IP, Topol EJ. Convergence of atherosclerosis and Alzheimer's disease: Cholesterol, inflammation, and misfolded proteins. Lancet 2004;363:1139-1146.

4. Ferro DA, Hilde VDB, Exalto LG, et al. Clinical relevance of acute cerebral microinfarcts in vascular cognitive impairment. Neurology 2019;92:e1558-e1566.

5. Karakis I, Pase MP, Beiser A, et al. Association of serum vitamin $\mathrm{D}$ with the risk of incident dementia and subclinical indices of brain aging: The Framingham Heart Study. J Alzhmers Dis 2016;51:451-461.

6. Cai HB, Wu GL, Huang ZS, et al. Effect of Zhuang Jing decoction on learning and memory ability in aging rats. Rejuvenation Res 2016;19:303-308.

7. Lan Z, Liu J, Chen L, et al. Danggui-Shaoyao-San ameliorates cognition deficits and attenuates oxidative stress- related neuronal apoptosis in d-galactose-induced senescent mice. J Ethnopharmacol 2012;141:386-395.

8. Sun YQ, Wang Q. Research progress of Danggui-Shaoyao San on treatment of gynecological diseases. J Clin Med 2017;4:12230.

9. Fu X, Wang QH, Wang ZB, et al. Danggui-Shaoyao-San: New hope for Alzheimer's disease. Aging Dis 2016;7:502.

10. Rossi R, Inzitari D, Pantoni L, et al. Nimodipine in subcortical vascular dementia trial. Alzheimer Dis Assoc Disord 1999;13:19.

11. Jong GID, Traber J, Luiten PGM. Formation of cerebrovascular anomalies in the ageing rat is delayed by chronic nimodipine application. Mech Ageing Dev 1992;64:255272.

12. Kanekiyo T, Bu G. The low-density lipoprotein receptorrelated protein 1 and amyloid- $\beta$ clearance in Alzheimer's disease. Front Aging Neurosci 2014;6:93.

13. Gaultier A, Arandjelovic S, Niessen S, et al. Regulation of tumor necrosis factor receptor-1 and the IKK-NF-kappa B pathway by LDL receptor-related protein explains the antiinflammatory activity of this receptor. Blood 2008;111: 5316-5325.

14. Xi Y, Wang M, Zhang W, et al. Neuronal damage, central cholinergic dysfunction and oxidative damage correlate with cognitive deficits in rats with chronic cerebral hypoperfusion. Neurobiol Learn Mem 2014;109:7-19.

15. Gupta S, Singh P, Mohan Sharma B, et al. Neuroprotective effects of agomelatine and vinpocetine against chronic cerebral hypoperfusion induced vascular dementia. Curr Neurovasc Res 2015;12:240-252.

16. Karin M. How NF-kappaB is activated: The role of the I kappa B kinase (IKK) complex. Oncogene 1999;18:68676874.

17. Karin M, Ben-Neriah Y. Phosphorylation meets ubiquitination: The control of NF- $\kappa \mathrm{B}$ activity. Annu Rev Immunol 2000;18:621-663.

18. Hyman BT, Yuan J. Apoptotic and non-apoptotic roles of caspases in neuronal physiology and pathophysiology. Nat Rev Neurosci 2012;13:395-406.

19. Li Z, Jo J, Jia JM, et al. Caspase-3 activation via mitochondria is required for long-term depression and AMPA receptor internalization. Cell 2010;141:859-871.

20. Erturk A, Wang Y, Sheng M. Local pruning of dendrites and spines by caspase-3-dependent and proteasome-limited mechanisms. J Neurosci 2014;34:1672-1688.

21. Lo SC, Wang Y, Weber M, et al. Caspase-3 deficiency results in disrupted synaptic homeostasis and impaired attention control. J Neurosci 2015;35:2118-2132.

22. Chen SX, Cherry A, Tari PK, et al. The transcription factor MEF2 directs developmental visually driven functional and structural metaplasticity. Cell 2012;151:41-55.

23. Ma X, Xu W, Zhang Z, et al. Salvianolic acid B ameliorates cognitive deficits through IGF-1/Akt pathway in rats with vascular dementia. Cell Physiol Biochem 2017;43:13811391.

24. Zhang T, Gu J, Wu L, et al. Neuroprotective and axonal outgrowth-promoting effects of tetramethylpyrazine nitrone in chronic cerebral hypoperfusion rats and primary hippocampal neurons exposed to hypoxia. Neuropharmacology 2017;118:137-147.

25. Chen L, Qi J, Chang YX, et al. Identification and determination of the major constituents in Traditional Chinese Medicinal formula Danggui-Shaoyao-San by HPLC-DADESI-MS/MS. J Pharm Biomed Anal 2009;50:127-137. 
26. Lana D, Melani A, Pugliese AM, et al. The neuronastrocyte-microglia triad in a rat model of chronic cerebral hypoperfusion: Protective effect of dipyridamole. Exp Gerontol 2014;6:46-62.

27. Himeno E, Ohyagi Y, Ma L, et al. Apomorphine treatment in Alzheimer mice promoting amyloid-beta degradation. Ann Neurol 2011;69:248-256.

28. Xie Z, Dong Y, Maeda U, et al. The common inhalation anesthetic isoflurane induces apoptosis and increases amyloid beta protein levels. Anesthesiology 2006;104:988-994.

29. Storck SE, Meister S, Nahrath J, et al. Endothelial LRP1 transports amyloid- $\beta 1-42$ across the blood-brain barrier. J Clin Invest 2016;126:123.

30. Kanekiyo T, Cirrito JR, Liu C-C, et al. Neuronal clearance of amyloid- $\beta$ by endocytic receptor LRP1. J Neurosci 2013; 33:19276-19283.

31. Liu CC, $\mathrm{Hu}$ J, Zhao N, et al. Astrocytic LRP1 mediates brain $\mathrm{A} \beta$ clearance and impacts amyloid deposition. J Neurosci 2017;37:4023-4031.

32. Kanekiyo T, Liu CC, Shinohara M, et al. LRP1 in brain vascular smooth muscle cells mediates local clearance of Alzheimer's amyloid- $\beta$. J Neurosci 2012;32:16458-16465.

33. Gaultier A, Salicioni AM, Arandjelovic S, et al. Regulation of the composition of the extracellular matrix by low density lipoprotein receptor related protein-1. J Biol Chem 2006;281:7332-7340.

34. Herz J, Strickland DK. LRP: A multifunctional scavenger and signaling receptor. J Clin Invest 2001;108:779-784.

35. Gorovoy M, Gaultier A, Campana WM, et al. Inflammatory mediators promote production of shed LRP1/CD91, which regulates cell signaling and cytokine expression by macrophages. J Leukoc Biol 2010;88.

36. Baud V, Karin M. Signal transduction by tumor necrosis factor and its relatives. Trends Cell 2001;11:372-377.

37. Aggarwal NT, Decarli C. Vascular dementia: Emerging trends. Semin Neurol 2007;27:66-77.

38. Erkinjuntti T, Roman G, Gauthier S, et al. Emerging therapies for vascular dementia and vascular cognitive impairment. Stroke 2004;35:1010-1017.

39. Kim JH, Ko PW, Lee HW, et al. Astrocyte-derived lipocalin-2 mediates hippocampal damage and cognitive deficits in experimental models of vascular dementia. Glia 2017;65:1471-1490.

40. Wyss-Coray T. Inflammation in Alzheimer disease: Driving force, bystander or beneficial response? Nat Med 2006; 12:1005-1015.

41. Lillis AP, Van Duyn LB, Murphy-Ullrich JE, et al. LDL receptor-related protein 1: Unique tissue-specific functions revealed by selective gene knockout studies. Physiol Rev 2008;88:887-918.

42. Cai H, Cong WN, Ji S, et al. Metabolic dysfunction in Alzheimers disease and related neurodegenerative disorders. Curr Alzheimer Res 2012;9:5-17.

43. Smith CJ, Lawrence CB, Rodriguez-Grande B, et al. The immune system in stroke: Clinical challenges and their translation to experimental research. J Neuroimmune Pharmacol 2013;8:867-887.

44. Liu Q, Zerbinatti CV, Zhang J, et al. Supplemental data amyloid precursor protein regulates brain apolipoprotein $\mathrm{E}$ and cholesterol metabolism through lipoprotein receptor LRP1. Neuron 2007;56:66-78.

45. Guojun B. Apolipoprotein E and its receptors in Alzheimer's disease: Pathways, pathogenesis and therapy. Nat Rev Neurosci 2009;10:333-344.
46. Yang L, Liu $\mathrm{C}-\mathrm{C}$, Zheng $\mathrm{H}$, et al. LRP1 modulates the microglial immune response via regulation of JNK and NF$\kappa \mathrm{B}$ signaling pathways. J Neuroinflammation 2016;13:304.

47. Marzolo MP, Von BR, Bu G, et al. Expression of alpha(2)macroglobulin receptor/low density lipoprotein receptorrelated protein (LRP) in rat microglial cells. J Neurosci Res 2000;60:401-411.

48. Hendrickx DA, Koning N, Schuurman KG, et al. Selective upregulation of scavenger receptors in and around demyelinating areas in multiple sclerosis. J Neuropathol Exp Neurol 2013;72:106.

49. Chuang TY, Guo Y, Seki SM, et al. LRP1 expression in microglia is protective during CNS autoimmunity. Acta Neuropathol Commun 2016;4:68.

50. Mantuano E, Brifault C, Lam MS, et al. LDL receptorrelated protein- 1 regulates NF- $\kappa \mathrm{B}$ and microRNA-155 in macrophages to control the inflammatory response. Proc Natl Acad Sci U S A 2016;113:1369-1374.

51. Overton CD, Yancey PG, Major AS, et al. Deletion of macrophage LDL receptorrelatedprotein increases atherogenesis in the mouse. Circ Res 2007;100:670-677.

52. Barnes PJ, Karin M. Nuclear factor- $\kappa$ B: A pivotal transcription factor in chronic inflammatory diseases. N Engl J Med 1997;336:1066-1071.

53. Karin M, Liu ZG, Zandi E. AP-1 function and regulation. Curr Opin Cell Biol 1997;9:240-246.

54. Kiyota T, Okuyama S, Swan RJ, et al. CNS expression of anti-inflammatory cytokine interleukin-4 attenuates Alzheimer's disease-like pathogenesis in APP+PS1 bigenic mice. FASEB J 2010;24:3093.

55. Saggu R, Schumacher T, Gerich F, et al. Astroglial NF- $\kappa B$ contributes to white matter damage and cognitive impairment in a mouse model of vascular dementia. Acta Neuropathol Commun 2016;4:76.

56. Hu Y, Ju SH, Zhang YJ, et al. Effect of Tongluo Xingnao effervescent tablets on learning and memory dysfunction in rats with chronic cerebral ischemia. Zhongguo Zhong Yao Za Zhi 2014;39:1908-1909.

57. El-Akabawy G, El-Kholy W. Neuroprotective effect of ginger in the brain of streptozotocin-induced diabetic rats. Ann Anat 2014;196:119-128.

58. Sodhi RK, Singh N. Defensive effect of lansoprazole in dementia of AD type in mice exposed to streptozotocin and cholesterol enriched diet. PLoS One 2013;8:e70487.

59. Newton K, Dixit VM. Signaling in innate immunity and inflammation. Cold Spring Harb Perspect Biol 2012;4:pii: a006049.

60. Serhan CN, Savill J. Resolution of inflammation: The beginning programs the end. Nat Immunol 2005;6:11911197.

61. Merry DE, Veis DJ, Hickey WF, et al. Bcl-2 protein expression is widespread in the developing nervous system and retained in the adult PNS. Development 1994;120:301-311.

62. Obulesu M, Lakshmi MJ. Apoptosis in Alzheimer's disease: An understanding of the physiology, pathology and therapeutic avenues. Neurochem Res 2014;39:2301-2312.

63. Wang HD, Fukuda T, Suzuki T, et al. Differential effects of Bcl-2 overexpression on hippocampal CA1 neurons and dentate granule cells following hypoxic ischemia in adult mice. J Neurosci Res 1999;57:1-12.

64. Zhang R, Xue YY, Lu SD, et al. Bcl-2 enhances neurogenesis and inhibits apoptosis of newborn neurons in adult rat brain following a transient middle cerebral artery occlusion. Neurobiol Dis 2006;24:345-356. 
65. Sun W. Programmed cell death of adult-generated hippocampal neurons is mediated by the proapoptotic gene Bax. J Neurosci 2004;24:11205-11213.

66. Ferrier A, De Repentigny Y, Lynch-Godrei A, et al. Disruption in the autophagic process underlies the sensory neuropathy in dystonia musculorum mice. Autophagy 2015;11:1025-1036.

67. Momeni HR, Soleimani-Mehranjani M, Shariatzadeh MA, et al. Caspase-mediated apoptosis in sensory neurons of cultured dorsal root Ganglia in adult mouse. Cell J 2013;15: 212-217.

68. Freeman LR, Keller JN. Oxidative stress and cerebral endothelial cells: regulation of the blood-brain-barrier and antioxidant based interventions. Biochim Biophys Acta 2012;1822:822-829.

69. Martindale JL, Holbrook NJ. Cellular response to oxidative stress: Signaling for suicide and survival. J Cell Physiol 2002;192:1-15.

70. Xu-Feng G, Chang-Jie C, Dong-Ping W, et al. Alteration of bcl-2 and bax expression in apoptosis of HL-60 cells induced by arsenic trioxide. J Bengbu Med Coll 2009;34: 9-11.

71. Zhang YL, Wei HL, Sun LJ. Impact of As2O3 on Bcl-2, survivin and reactive oxygen species (ROS) expressions during the process of induced apoptosis in multidrugresistant human leukemia K562/ADM cells. China Tumor 2008; 17:495-498.

72. Tian T. Studies on the mechanism of oxidative stress and Bcl-2 in HEK293 cells apoptosis induced by cadmium. Nan Jing: Submitted to Nanjing Normal University 2007;10:1192-1193.

73. Yue Z, Jun L, Neng-Hui H, et al. Protective effects and mechanism of Ganoderma lucidum triterpenoids on learning and memory function of Alzheimer disease model animals. J Food Sci Biotechnol 2012;31:741-774.

74. Liu RL, Liu IY, Bi XN, et al. Reversal of age-related learning deficits and brain oxidative stress in mice with superoxide dismutase/catalase mimetics. Proc Natl Acad Sci U S A 2003;100:8526-8531.

75. Román GC. Vascular dementia: Distinguishing characteristics, treatment, and prevention. J Am Geriatr Soc 2003; 51(5s2):S296-S304.

Address correspondence to: Qi Wang Institute of Clinical Pharmacology Guangzhou University of Chinese Medicine Guangzhou 510405

China

E-mail: gzzyydx@yahoo.com

Huafeng Pan Institute of Clinical Pharmacology Guangzhou University of Chinese Medicine Guangzhou 510405

China

E-mail: gzphf@126.com

Zhouke Guo Department of Neurology \& Psychology Shenzhen Traditional Chinese Medicine Hospital Fourth Clinical Medical College of Guangzhou University of Chinese Medicine Shenzhen 518033

China

E-mail: szszyy@yahoo.com

Received: February 26, 2019 Accepted: April 2, 2020 